\title{
Secession-Proof Cost Allocations and Stable Group Structures in Models of Horizontal Differentiation
}

\author{
Michel Le Breton* Shlomo Weber ${ }^{\dagger}$
}

September 2003

\section{Table of Contents}

Section 1. Introduction - page 2.

Section 2. General Model - page 5.

Section 3. Cooperative Framework - page 7.

Section 4. Unrestricted Cost Allocation Correspondence - page 9.

Section 5. Restricted Cost Allocation Correspondence - page 15.

Subsection 5.1. T-stability - page 16 .

Subsection 5.2. Individual T-stability - page 18 .

Subsection 5.3. Equal Cost Share Rule - page 20.

Section 6. Appendix - page 24.

Section 7. References - page 27.

\footnotetext{
${ }^{*}$ Université de Toulouse I, GREMAQ and IDEI, Toulouse, France.

${ }^{\dagger}$ Department of Economics, Southern Methodist University, Dallas, USA, and CORE, Catholic University of Louvain-la-Neuve, Belgium.
} 


\section{Introduction}

In many social, political and economic situations individuals form groups rather than operate on their own. For example, communities are formed in order to share the costs of production of local public goods among the residents, or workers join a labor union in order to attain a better working contract. In these situations individuals utilize the increasing returns to scale provided by large groups. On the other hand, given the heterogeneity of agents' characteristics and tastes, the decision-making process of a large group may lead to outcomes quite undesirable for some of its members. This observation supports the claim that benefits of size are not unlimited and, on some occasions, a decentralized organization is superior to a large social structure. Thus, instead of a grand coalition containing the entire population, we may observe the emergence of group structures which consist of groups smaller than the grand coalition. The reason for the existence of groups that contain more than one agent but less than the entire society lies in the conflict between increasing returns to scale on one hand and heterogeneity of agents' preferences on the other (see Chapter 6 by G. Demange in this volume).

In this chapter we consider a society which consists of a finite or infinite number of individuals. who are allowed to create subsocieties which lead to a partition of the society into pairwise disjoint groups. Each group in the partition selects an alternative within a policy space available to this group. The notion of policy (or project) in this chapter is similar to that of public project introduced by Mas-Colell (1980). It can accommodate virtually any interpretation, as long as the benefit derived by an agent from the selected project within the given group is not affected by the composition of the other members of this group.

The two basic elements of group formation described here, namely, the composition of formed groups and their policy choices do not provide, however, the complete description of the collective decision problem. What is missing here is a mechanism of sharing the policy costs among agents within the same group that choose the same policy. Indeed, a government has to select an explicit scheme to tax its citizens in order to cover the cost of public policies, whereas a community 
must determine the tax burden of its residents for financing local public projects. Thus, when a partition of individuals has been formed a set of appropriate policies has been chosen, the allocation of the cost among the agents should be determined.

Suppose now that the partition of individuals into groups has been formed, where each group in the partition chooses a policy, and finally, each group decides on allocation of policy costs among its members. For an arbitrarily chosen cost allocation, however, there may exist a single individual or a group of agents who would object the proposed arrangement. A detailed analysis of "secession" threats is provided and would depend on specific stability notions, but to illustrate the point, there could be a group of agents that can reject arrangement by choosing a policy and a device for financing it such that every member of the "seceding" group would be better off compared to the current arrangement.

It is important to point out a clear separation between "horizontally differentiated" and "vertically differentiated" projects. If the interpretation of the policy space is given by a choice of geographical location of public facilities (schools, hospitals, libraries, etc.), then the individuals display distinct preferences over the policy space and the location turns to be the parameter of horizontal differentiation. If, on the other hand, the agents exhibit identical preferences over quantity or quality attributes of public projects, we will have a model of "vertical differentiation". 1 In this chapter we focus mainly on models with horizontal differentiation where individuals have different rankings over elements of policy space. ${ }^{2}$

In examining collective decisions and corresponding cost allocations, our main focus is the study of "secession-proof" allocations that are immune against individual or group deviations from the proposed arrangement. When analyzing possible cost allocations, we have to take into account that in many cases a variety of institutional, legal, information, and technological constraints may rule out some cost allocation schemes for certain groups of individuals. We therefore will

\footnotetext{
${ }^{1}$ The general framework developed in this chapter allows for the examination of the existence and structure of Tiebout equilibria. Our approach is, by no means, exclusive. For example, economies with local public goods and economies with clubs have been analysed within the Arrow-Debreu general equilibrium framework (see, e.g., Conley and Wooders (1997), Ellickson, Grodal, Scotchmer and Zame (1999) and Wooders $(1978,1980,1988)$.

${ }^{2}$ The case of vertical differentiation is discussed in Chapter 6 by G. Demange.
} 
distinguish between two cases:

A - unrestricted set of cost allocations. In this case there are no restrictions on the set of cost allocations, so that every group that forms or contemplates its formation is allowed to use any cost sharing scheme among its members. The only condition is that the aggregated individual contributions of the members of the group cover the cost of their chosen policies.

B - restricted set of cost allocations. Here some groups face institutional or social limitations on the sets of possible cost allocations they can use. We examine several specific cost sharing schemes such as:

Equal Share allocations - all individuals within the same group, regardless of their characteristics and policy preferences, make equal contribution towards policy costs;

Rawlsian allocations - the cost allocations that are designed to equalize the after-contribution utilities and eliminate the utility gaps among all members of the given group. In this chapter we do not examine the issue of feasibility of public projects which can have many different interpretations. It may incorporate constraints that are generated by institutions, rules or social norms. If the "projects" under consideration are allocations of goods, feasibility, as usual, means that, due to resource constraints, some alternatives that are available for some groups could be out of reach for some others. One case is that of increasing returns when larger groups have an access to larger sets of projects. For example, a merger of two groups may create a pool of resources that was not available in the pre-merger state. Obviously, one can imagine the situations when the opposite is true in the case when the chances to reach an acceptable solution for all members would diminish in large group. All these situations are beyond the scope of our analysis here. ${ }^{3}$

In this chapter we simply assume that all coalitions can choose any project from the entire policy space. It is quite natural to expect that the existence of stable structures is crucially dependent on the dimensionality of the policy space. In general, the severity of preferences' divergence raises when the number of policy dimensions increases, in which case the search for

\footnotetext{
${ }^{3}$ A reader can be referred to Greenberg and Weber (1993), Le Breton and Weber (2003b,c) for the study on variable feasible sets.
} 
a stable group structure and an appropriate cost share allocation becomes more challenging. We will have focus here on unidimensional policy spaces which will allow us to obtain a large set of interesting results and in the same time to indicate the difficulties that may arise in the multidimensional setting.

\section{General Model}

We introduce the description of a society with one private good which plays the role of numeraire. Formally, we consider a society $\mathcal{E}$ which is defined by a sextuple $(N, X, C, T, W, U)$, where

$N$ is a set of individuals,

$X$ is a set of projects,

$C$ is a cost correspondence that determines the cost (in terms of numeraire) incurred by a coalition $S$ when it selects a project $x \in X$,

$T$ is a cost share correspondence that assigns the set of feasible cost share allocations for every coalition $S$ and every project $x \in X$,

$W$ is a profile of initial endowments (incomes) $\{w(i)\}_{i \in N}$ in terms of numeraire,

$U=\left\{u_{i}\right\}_{i \in N}$ is a profile of individuals' utility functions that represent their preferences over pairs of public projects and a consumption of private good $u_{i}: X \times \Re_{+} \rightarrow \Re_{+}$.

Now let us turn to a more detailed description of all components that constitute a society.

The set of agents $N$. We consider both finite and infinite sets of individuals. In the infinite case we consider societies where the weight of any individual is negligible; such societies will be referred to as being "nonatomic" or without "atoms". The set of individuals $N$ in such a setting is represented by the measure space $\{N, \mathcal{S}, \lambda\}$, where $\lambda$ is an atomless measure and $\mathcal{S}$ is a set of nonnegligible measurable subsets of $N$. A canonical example, that will be used throughout this chapter, is the case of the unit interval, where $N=[0,1], \mathcal{S}$ is the Borel $\sigma$-algebra of $[0,1]$ and $\lambda$ is the Lebesgue measure on $[0,1]$.

In the case of finite societies we consider $\lambda$ as the counting measure and $\lambda(S)$ or, simply $|S|$ 
will stand for the cardinality of the set $S$. This assumption captures situations which underline the importance of actions of individuals. We refer to these societies as being atomic. In such a case we will denote by $n$ the number of individuals, i.e., $N=\{1, \ldots, n\}$ and $\mathcal{S}$ will simply denote the collection of all nonempty subsets of $N$.

Policy space $X$. Again we consider both cases, of finite and infinite policy space $X$. For most of the paper we will assume that the set $X$ is finite. However, in some cases it is more appropriate to consider an infinite set $X$. We then assume, as we indicated above, that $X$ is a convex and compact subset of the unidimensional set $\Re$. Note that even though convexity is a very natural assumption in many applications, it may not apply in the first best environments with basic non convexities. The second best constraints may also distort the convex structure of environments created by first best constraints.

Every group of individuals that forms, can choose an alternative from $X$. Even though some policies, due to resource constraints, institutional rules or social norms, could be out of reach for some coalitions, we do not consider these possibilities here and assume, as in the vast majority of contributions in this research area (with the exception of Greenberg and Weber (1993) and Le Breton and Weber $(2003 \mathrm{~b}, \mathrm{c}))$, that all policies in $X$ are feasible for all coalitions.

Cost correspondence $C$. The cost correspondence $C: X \times \mathcal{S} \rightarrow \Re_{+}$, that determines the cost (in terms of numeraire) incurred by coalition $S$ when it selects the public project $x$. In our framework the cost of every project assumes the separable form: $C(S, x)=\tilde{C}(S)+c(x)$. On many occasions the function $\tilde{C}(S)$ is an increasing function of of the mass of members of $S$. This would imply that the group-specific component of the cost of the public project increases with the size of the group to be served by this project. Since we deal with the horizontal differentiation framework, our focus is on distinct preferences over the set of projects and we do not examine here the cost differentials across projects. Then we often assume that the project-specific component of the project cost, $c(x)$, is independent of $x$ and $c(x)=c$ for all projects $x$.

Cost allocation correspondence $T$. For every coalition $S \in \mathcal{S}$ and a project $x \in X$, any cost sharing rule should guarantee that the aggregated contributions of members of $S$ cover the cost of 
the project $C(S, x)$. Formally, a measurable function $t$ on $S$ is a (balanced) cost allocation if the total aggregated contribution of members of $S$ is equal to $C(S, x)$. The set of all cost allocations for $S$ and $x$ is denoted by $T^{*}(S, x)$.

As mentioned above, due to possible institutional, technological or other type of constraints, the cost allocation correspondence $T$ assigns to every coalition $S$ and every project $x$ the set of feasible cost allocations $T(S, x)$, which could be a smaller set that the set of balanced cost share allocations $T^{*}(S, x)$. If the cost allocation correspondence is single-valued, then for $i \in S$, the value $T(i, S, x)$ will denote the cost share of individual $i$ within $S$ used to finance the project $x$. Otherwise, for every cost allocation $t \in T(S, x)$ we denote by $t(i)$ the cost share of individual $i$.

Profile of initial endowments. Each individual $i \in N$ is endowed with a positive amount of the private good $w(i)$. Then $W=\{w(i)\}_{i \in N}$ is the aggregate profile of the initial endowments.

Profile of individuals' utility functions $U$. A profile of utility functions $\left.u_{i}(\cdot, \cdot)\right)_{i \in N}$, where $u_{i}: X \times \Re_{+} \rightarrow \Re$ represents the preferences of individual $i$ over all bundles $\left(x, m_{i}\right)$, consisting of a public project $x$ and a consumption of private good $m(i)$. We impose two basic assumptions on all function $u_{i}$. First, we assume that, for any given project $x$, every function $u_{i}(x, \cdot)$ is increasing with respect to the amount of private good. Secondly, in the models with the infinite set of projects $X$, for a given amount of private good, the utility function of every individual is quasi-concave in policy choices. In some of our examples we assume that that the functions $u_{i}$ are quasi-linear, i.e., $u_{i}\left(x, m_{i}\right)=h_{i}(x)+m_{i}$. In this case the function $h_{i}(\cdot)$ is quasi-concave.

\section{Cooperative Framework}

Let us now turn to the cooperative approach of the group formation process and associate a society with a cooperative game in characteristic function form. To avoid the introduction of the heavy machinery required for the treatment of societies with the infinite number of individuals, we provide the formal definition of the game for a case with a finite number of individuals.

Let the cost allocation correspondence $T$ be given. We then associate $\mathcal{E}$ with a cooperative game in characteristic form $v^{T}$, where for each coalition $S \subset N$, the set of utility levels attainable 
for the members $S$ is determined. That is, a utility vector $u=\left(u_{1}, \ldots, u_{n}\right)$ is attainable for $S$ if there exists a public project $x \in X$ and a cost share allocation $t \in T(S, x)$ such that every member of $i \in S$ achieves at least the utility level $u_{i}$. Formally,

$$
v^{T}(S)=\left\{u=\left(u_{1}, \ldots, u_{n}\right) \in \Re^{n}: \exists x \in X \text { and } t \in T(S, x) \text { s. t. } u_{i} \leq u_{i}(x, w(i)-t(i)) \forall i \in S\right\} .
$$

If the sets of cost share allocations are unrestricted, the game $v^{T^{*}}$ will be simply denoted by $v$.

We first introduce the standard definition of the core:

Definition 3.1: A payoff vector $u=\left(u_{1}, \ldots, u_{n}\right) \in \Re^{n}$ is in the core of the game $v^{T}$ if $u \in v^{T}(N)$ and there is no coalition $S \subseteq N$ and $u^{\prime}=\left(u_{1}^{\prime}, \ldots, u_{n}^{\prime}\right) \in v^{T}(S)$ such that $u_{i}^{\prime}>u_{i}$ for all $i \in S$.

As we show below, the cooperative game $v^{T}$ is not necessarily superadditive, i.e., it is possible that there exist coalitions $S, S^{\prime} \subset N$ for which the inclusion $v^{T}(S) \bigcap v^{T}\left(S \backslash S^{\prime}\right) \subset v^{T}(S)$ does not hold. This means that there could be two disjoint coalitions such that their union does not necessarily guarantee all the members of two groups the utility levels they could achieve separately. To rectify this situation, we define the cooperative game $\widehat{v}^{T}$, which is the superadditive cover of the game $v^{T}$. Denote by $\mathcal{P}$ the set of all partitions or group structures ${ }^{4}$ of $N$ and consider a partition $P=\left\{S^{k}\right\}_{1 \leq k \leq K} \in \mathcal{P}$. This partition yield the set of feasible payoff vectors given by the following intersection:

$$
v^{T}(P)=\cap_{1 \leq k \leq K} v^{T}\left(S^{k}\right)
$$

Then $\widehat{v}^{T}$ is defined by

$$
\widehat{v}^{T}(S)=\cup_{P_{S} \in \mathcal{P}(S)} \cap_{S^{\prime} \in P_{S}} v^{T}\left(S^{\prime}\right)
$$

for all $S \subseteq N$, where $\mathcal{P}(S)$ denotes the set of partitions of $S$. The game $\widehat{v}^{T}$ describes the set of payoffs attainable for coalitions assuming they can be partitioned in any arbitrary way. It is easy to verify that the games $v^{T}$ and $\widehat{v}^{T}$ coincide if and only if the latter is superadditive.

\footnotetext{
${ }^{4}$ Cooperative games with a coalition structure were introduced by Aumann and Drèze (1974). See also Greenberg (1994).
} 
Before introducing the notion of a $T$-stable outcome, we should point out that a $T$-attainable outcome in the society consists of three elements: partition of the set of individuals in pairwise disjoint groups, public projects in $X$ and feasible (given $T$ ) cost sharing allocations chosen by each group in the formed partition. Formally:

Definition 3.2: The set of triples $z=\left\{\left(S^{k}, x^{k}, t^{k}\right)\right\}_{1 \leq k \leq K}$ is called a T-attainable outcome, where $\left\{S^{k}\right\}_{1 \leq k \leq K}$ is a partition of the set of individuals $N$ into pairwise disjoint groups; $\left(x^{1}, \ldots, x^{K}\right)$ is a set of public projects with $x^{k} \in X$ being chosen by $S^{k}$ for all $k=1, \ldots, K$; $\left(t^{1}, \ldots, t^{K}\right)$ is a set of cost sharing allocations with $t^{k} \in T\left(S^{k}, x^{k}\right)$ being chosen by $S^{k}$ for all $k=1, \ldots, K$.

From the cooperative point of view, a $T$-attainable outcome $z$ is stable if it is immune against all coalitional deviations. In other words, the utility vector associated with $z$ belongs the core of the corresponding game. Since the game $v^{T}$ is not, in general, superadditive, we allow multi-group configurations and, following Guesnerie and Oddou $(1979,1981)$, we consider the game $\widehat{v}^{T}$ instead of $v^{T}$ :

Definition 3.3: A $T$-attainable outcome $z=\left\{\left(S^{k}, x^{k}, t^{k}\right)\right\}_{1 \leq k \leq K}$ is $T$-stable if the utility vector $u=\left(u_{1}, \ldots, u_{n}\right)$, where $u_{i}=u_{i}\left(x^{k(i)}, w(i)-t(i)\right)$ for every $i \in S^{k(i)}$, belongs to the core of $\widehat{v}^{T}$. A $T^{*}$-stable outcome will be simply called stable.

If the outcome $z$ is $T$-stable, then, given the partition of the set $N$ and the choice of public projects, the contribution scheme $\left(t_{1}, \ldots, t_{n}\right)$, associated with $z$, is immune against any threat of coalitional deviations. This scheme will be called T-secession-proof. A $T^{*}$ secession-proof scheme is simply secession-proof.

In the next section we study the existence of stable outcomes and provide a partial characterization of secession-proof allocations. In light of the discussion of this section, we proceed by examining the core of the game $\widehat{v}^{T^{*}}=\widehat{v}$. 


\section{Unrestricted Cost Allocation Correspondence}

As in Cremer, De Kerchove and Thisse (1985), Alesina and Spolaore (1997), Le Breton and Weber (2003a), Haimanko, Le Breton and Weber (2003a,b), we consider a society that consists of agents with preferences over the set $X$ of possible locations of public projects, where the set $X$ is given by the interval $[0,1]$. Every agent $i \in N$ has an ideal point $p^{i}$ in $X$ and the preferences that are symmetric and single-peaked, so we can identify $i$ with her ideal point in $X$. The distribution of ideal points is given by a cumulative distribution function $F$, defined over the space $X$. It is important to stress that for our main existence result in this section we do not impose any restrictions on the distribution function and allow for infinite or finite societies. We denote by $\mu$ the measure on $X$ induced by the distribution function $F$ with the total mass $\mu(X)$ equal to 1 .

Every (measurable) group of individuals $S$ can choose any location $x \in X$. The costs associated with any project $x$ for the community $S$ are given by $C(S, x)=\alpha \mu(S)+c(x)$.

The project cost consists of two components: the variable cost, $\alpha \mu(S)$, that depends on the number of individuals in $S$ (project users), where $\alpha$ is a nonnegative constant, and the positive fixed cost of setting and maintaining the project, $c(x)$. We assume that $c(\cdot)$ is a continuous and positive-valued function on $X$. In this section we do not restrict the set of balanced cost share allocations, and for every measurable subset $S$ of $N$ and every $x \in X$, the set of feasible cost share allocations, $T^{*}(S, x)$, consists of all measurable functions $t$ defined on the set $S$, satisfying $\int_{S} t(i) d \mu(t)=C(S, x)$.

If an individual $i$ belongs to the group $S$ that chooses project $x \in X$, she incurs a disutility or "transportation" cost, $d(i, x)$, which is determined by the distance between $i$ and the location $x$ of the project. We only require that the cost function $d(i, x)=d(|i-x|)$ is continuous and (strictly) increasing in the distance $|i-x|$, with $d(0)=0$.

The utility of member $i$ of group $S$ that chooses project $x$ and the cost share allocation $t$ consists of two terms, the transportation cost $-d(i, x)$ and her net income $w(i)-t(i)$. In our separable setting the initial endowment would not impact individuals' decisions and we simply 
ignore it. Thus, without loss of generality, the utility of individual $i$ is given by $u_{i}\left(x, m_{i}\right)=$ $-d(i, x)-t(i)$.

Definition 3.3 implies that in this framework a stable outcome exists if and only if the game $\widehat{v}$, where the set $v(S)$ is given by:

$$
v(S)=\left\{u=\left(u_{1}, \ldots, u_{n}\right) \in \Re^{n}: \exists x \in X \text { and } t \in T^{*}(S, x) \text { s. t. } u_{i} \leq-d(i, x)-t(i) \forall i \in S\right\}
$$

for every group $S$, has a nonempty core. We have the following existence result:

Proposition 4.1 (Haimanko, Le Breton and Weber (2003a)): A stable outcome exists for all finite and infinite societies.

Haimanko, Le Breton and Weber (2003a) also show that if the society is nonatomic and is described by a strictly positive and continuous density, then, given the costs $c$, for any stable outcome and any interval $S \subseteq N$, the number of groups in $S$, denoted $K(c, S)$, satisfies the following asymptotic formula called the square root principle:

$$
\lim _{c \rightarrow 0} \sqrt{c} K(c, S)=\frac{\sqrt{d_{+}^{\prime}(0)}}{2} \int_{S} \sqrt{f(t)} d t,
$$

where $d_{+}^{\prime}(0)$ is the (well-defined) right-hand side derivative of the function $d$ at 0 . The square root principle implies that if $c$ is small, the asymptotic ratio of the number of groups in intervals $S$ and $T$ is given by $\frac{\int_{S} \sqrt{f(t)} d t}{\int_{T} \sqrt{f(t)} d t}$. Note this ratio is different from the population ratio $\frac{\int_{S} f(t) d t}{\int_{T} f(t) d t}=\frac{\lambda(S)}{\lambda(T)}$, unless $\lambda$ is generated by the uniform distribution!

Proposition 4.1 guarantees the existence of stable partitions under appropriate choice of projects and cost share allocations but it does not provide a characterization of the policy choices and, especially, of cost allocation secession-proof schemes. However, the issue of redistribution of economic costs across various groups has important theoretical and practical implications in many contexts, including the study of country formation and redistributive policies that aim at preventing conflicts within countries with heterogeneous population. There are several sources of population heterogeneity, that may cause dissatisfaction of significant groups of the society with 
the one-size-fits-all choices made by the central or regional governments. They include country's geographic structure, attitudes towards protection of minority rights, promotion and preservation of distinctive local culture and language, disagreement over spending policies, the country cultural, ethnical, religious and language fractionalization, economic regional disparity, etc. With sufficient heterogeneity and diversity of the country's population, government's inability to satisfy all the preferences in the country is bound to breed dissatisfaction within certain group and regions, thereby creating a potential for internal conflicts and threats of secession. Since usually, the consequences of conflicts (political instability, inadequate economic performance, etc.) are undesirable from the countrywide perspective, many countries have explicit transfers schemes to assist their disadvantaged regions. ${ }^{5}$ Since the existence of redistribution schemes has been examined in our framework by Proposition 4.1, the natural question that remains is what is a set of policy instruments available to the government in order to mitigate secessionist sentiments.

Suppose now through the rest of the section that the project-specific part of the cost $c(x)$ is project independent, i.e., the costs associated with any project $x$ for the community $S$ are given by $C(S, x)=\alpha \mu(S)+c$, where $c$ is a positive constant. We also assume that the transportation costs are linear, i.e. $d(i, x)=|i-x|$ for all $i \in N$ and all $x \in X$.

Since a seceding region has to incur substantial costs of laying foundations for the new country and running its administration, the breakaway tendencies are countered by the economies of scale generated by being a united political entity. In the case where the secession costs are too high, the stable outcome allows no partitions of the grand coalition.

Proposition 4.2 (Haimanko, Le Breton and Weber (2003b)): There exists a value $\bar{c}$ such that a society admits a stable outcome consisting of the triple $\{N, x, t\}$, where $x \in X$ and $t \in T^{*}(N, x)$, if and only if $c \geq \bar{c}$. For a given distribution of individuals' ideal points $F$, we denote $c^{F}=\bar{c}$.

Consider now the nonatomic societies represented by the atomless distribution over the interval

\footnotetext{
${ }^{5}$ The examples include Russia, China, France, Italy, Belgium, Germany, Canada, Australia and many other countries. See Ter-Minassian (1997), Ahmad and Craig (1997), Le Breton and Weber (2003a).
} 
$X=[0,1]$. We impose two assumptions on the cumulative distribution functions to be used in our analysis. First is quite standard:

SY - Symmetry: The density function $f$ is symmetric with respect to $\frac{1}{2}$, the center of $X$, i.e., $f(i)=f(1-i)$ for all $i \in X$.

To introduce the second assumption, for $i \in X$, denote by $L_{i}=[0, i]$ and $R_{i}=[i, 1]$. Let $l_{i}$ and $r_{i}^{6}$ will be their respective median locations, i.e., $F(l(i))=\frac{1}{2} F(i)$ and $1-F(r(i))=\frac{1}{2}(1-F(i))$.

Note that $r(i)=1-l(1-i)$ for every $i \in X$

We assume that the functions $l$ and $r$ are differentiable except possibly in a finite number of points. We assume:

GEM - Gradually Escalating Median: The derivative $l^{\prime}(i)$ satisfies $l^{\prime}(i) \leq 1$ on the interval $[0,1]$. Obviously, the symmetry assumption would imply $r^{\prime}(t) \leq 1$.

We denote by $\mathcal{F}$ the set of distribution functions satisfying the symmetry and $G E M$ assumptions.

Assumption $G E M$ implies that if we increase the length of the interval $L_{i}=[0, i]$ by a small positive number $\delta$, then the median of the interval $L_{i+\delta}=[0, i+\delta]$ is shifted to the right by the increment smaller than $\delta$.

The class of distribution functions satisfying GEM contains the family of log-concave functions. That class, in turn, includes "truncated" versions of the uniform, the normal and the exponential distributions. ${ }^{7}$ There are, in addition, distribution functions that are not log-concave but nevertheless satisfy the GEM assumption, such as some classes of bimodal distributions. ${ }^{8}$

For every distribution function $F$ that belongs to the class $\mathcal{F}$, the threshold value $c^{F}$, defined in Proposition 4.2, has been explicitly derived in Haimanko, Le Breton and Weber (2003b):

Proposition 4.3: For every $F \in \mathcal{F}$

$$
c^{F}=\frac{1}{2}-4 \int_{l\left(\frac{1}{2}\right)}^{\frac{1}{2}} i f(i) d i,
$$

\footnotetext{
${ }^{6}$ If $f$ is positive everywhere, then the functions $l$ and $r$ are well-defined. Otherwise, the median locations are not not determined uniquely and constitute an interval. In this case we take "median" as the midpoint of this interval.

${ }^{7}$ See Bagnoli and Bergstrom (1989), Caplin and Nalebuff (1991), Weber (1992).

${ }^{8}$ See Le Breton and Weber (2003a).
} 
where $f$ is the density function of the cumulative distribution $F$. If the distribution $f$ is uniform on $[0,1]$, then $c^{F}=\frac{1}{8}$.

We now turn to a characterization of stable outcomes and secession-proof cost allocation. First we know that if the project $\operatorname{cost} c$ is greater or equal to $c^{F}$, then the grand coalition $N$ should form. The symmetry of the distributions we consider makes it natural to consider the location of the public project $x$ at the geographical center of $X, \frac{1}{2}$. To examine secession-proof allocations consider the following cost share allocation in $N$ :

$$
t^{c}(i)= \begin{cases}r(i)+\beta & \text { if } \quad i \leq \frac{1}{2} \\ r(1-i)+\beta & \text { if } \quad i \geq \frac{1}{2}\end{cases}
$$

where $\beta$ is chosen to satisfy the budget constraint: $\beta=c-\int_{0}^{1} t^{c}(i) f(i) d i$.

Proposition 4.4 (Le Breton and Weber (2003a)): Let $F \in \mathcal{F}$ be given. If the project cost $c$ satisfies $c \geq c^{F}$, the triple $\left\{N, \frac{1}{2}, t^{c}\right\}$ is a stable outcome. That is, the cost allocation $t^{c}$ is secession-proof.

Note that the function $t^{c}$ is symmetric around the the center $\frac{1}{2}$, is increasing on the interval $\left[0, \frac{1}{2}\right]$ and is decreasing on the interval $\left[\frac{1}{2}\right]$. That implies that the closer an individual to the center the larger her cost share is. Thus, individuals who are located close to the endpoints are subsidized by those located closer to the center. However, since the GEM implies the total contribution (including the transportation cost), given by $\left|i-\frac{1}{2}\right|+t^{c}(i)$ is still higher for those close to the endpoints. Thus, this allocation satisfies the principle of partial equalization, where while some equalization takes place, it is not full. It is interesting to note that in the case of the uniform distribution the equalization rate is $50 \%$.

It is important to stress that two important allocations often studied in the literature are not necessarily secession-proof when $c \geq c^{F}$. Consider the uniform distribution $F$ whose density $f(i)$ is equal to 1 for all $i$ on interval $[0,1]$. First, consider an equal share allocation $t^{E}$ under which all individuals contribute the same amount $t^{E}(i)=c$ towards the project costs. Secondly, examine the Rawlsian allocation $t^{R}$ that guarantees the equal total contribution (including transportation 
cost) for all individuals. That is, $t^{R}(i)+\left|i-\frac{1}{2}\right|$ should be the same for all $i \in N$. It is easy to derive:

$$
t^{R}(i)=\left\{\begin{array}{lll}
i-\frac{1}{4}+c & \text { if } & i \leq \frac{1}{2} \\
-i+\frac{3}{4}+c & \text { if } & i \geq \frac{1}{2}
\end{array}\right.
$$

Then:

Remark 4.5 (Le Breton and Weber (2003a)): Let $F$ be the uniform distribution on the interval $[0,1]$. Then

(i) There exists $c^{*}>\frac{1}{8}$ such that for every $c, \frac{1}{8} \leq c<c^{*}$, the triple $\left\{N, \frac{1}{2}, t^{E}\right\}$ is not a stable outcome. That is, the equal cost share allocation $t^{E}$ is not, in general, secession-proof.

(ii) For every $c, \frac{1}{8} \leq c<\frac{1}{4}$, the triple $\left\{N, \frac{1}{2}, t^{R}\right\}$ is not a stable outcome. That is, the Rawlsian cost share allocation $t^{E}$ is not, in general, secession-proof.

In the case of the equal cost share allocation $t^{E}$, the individuals in the center do not provide equalization transfers to those close to the endpoints. Thus, there would be coalitions of distant individuals who may reject the proposed cost share. Indeed, there are coalitions $[0, i]$ with $\frac{1}{3}<$ $i<\frac{1}{2}$ that would be better off on their own. Note that the seceding coalition should be sufficiently large to mitigate the increasing returns effect generated by the grand coalition $N$.

In the case of the Rawlsian cost share allocation $t^{R}$, the individuals close to the center carry the major burden of equalization transfers to those close to the endpoints. Thus, there are coalitions of individuals in the center who would rather create their own group than fully subsidizing the rest of the society.

We now turn to the case where some coalitions are prevented from using all balanced cost share allocations to finance public projects.

\section{Restricted Cost Allocation Correspondence}

In this section we examine an extreme case where the cost allocation correspondence $T$ is single-valued. That is, for every coalition $S$ and a public project $x \in X$, the coalition $S$ possesses only one scheme to share the cost of a project $x \in X$ among its members. In this 
case every individual $i \in S$ contributes an amount $T(i, S, x)$ towards the project cost. It allows us to introduce the indirect utility function $v_{i}(S, x): \mathcal{S}^{i} \times X \rightarrow \Re$ for every individual $i$, where $\mathcal{S}^{i}$ is the set of all coalitions that contain $i$. This function is defined as follows: $v_{i}(S, x)=$ $u_{i}(x, w(i)-T(i, S, x))$.

We impose the following important assumption that will be satisfied through the rest of the chapter.

Assumption PE - Positive Externality: The inequality $v_{i}(S, x)<v_{i}\left(S^{\prime}, x\right)$ holds for every individual $i \in N$, every project $x \in X$ and every two different coalitions $S, S^{\prime} \in \mathcal{S}^{i}$ and $S \subset S^{\prime}$.

This assumption implies that if one or several individuals join the existing coalition, they would have a positive effect on the utility of existing members of the group. In the case of separable utilities it simply means that the monetary burden of financing a given project would be reduced in a larger group. Note that the $P E$ assumption holds when, in particular, the cost of public projects is constant and the society uses either the equal cost share correspondence $T^{E}$ or the tax proportional rule, where each individual contributes the same proportion of her initial endowment.

In the next subsection we examine the issue of $T$-stability for the environments satisfying $P E$.

\section{$5.1 \quad T$-stability}

Since the utility functions of all individuals depend only on the project and the coalition the individual $i$ belongs to, one can reduce Definition 3.2 of a $T$-stable outcome to a choice of a partition and corresponding public projects. The corresponding cost share allocations would be uniquely determined by the single-valued cost allocation correspondence. Our first results in this framework are obtained when either the set of public projects $X$ consists of two elements, as in many models with network externalities (Arthur (1989), Farrell and Saloner $(1985,1988)$, Tirole (1988)), or set $N$ contains two individuals. In both cases the existence of a $T$-stable outcome is guaranteed by $P E$ : 
Proposition 5.1.1 (Konishi, Le Breton and Weber (1997a): Let $|X|=2$. Then under PE, society $\mathcal{E}$ admits a $T$-stable outcome.

Proposition 5.1.2 (Konishi, Le Breton and Weber (1997b)): Let $|N|=2$. Then, under $P E$, society $\mathcal{E}$ admits a $T$-stable outcome.

Propositions 5.1.1 and 5.1.2 cannot be extended to the case where the set of alternatives and set of individuals each contain more than two elements. Before describing the example showing that this is indeed the case, let us introduce the following assumption:

Assumption AN - Anonymity: The equality $v_{i}(S, x)=v_{i}\left(S^{\prime}, x\right)$ holds for every individual $i \in N$, every project $x \in X$ and every two coalitions $S, S^{\prime} \in \mathcal{S}^{i}$ with $|S|=\left|S^{\prime}\right|$. If the society satisfies $A N$, we will write, without abuse of notation, $v_{i}(|S|, x)$ instead of $v_{i}(S, x)$.

This assumption holds under the equal share cost allocation. However it would not, in general, be satisfied if the society adopts the tax proportional rule. In this case every individual would be better off in the same size group with wealthier individuals rather than with poorer ones.

The following example, taken from Konishi, Le Breton and Weber (1997a), shows that a Tstable outcome may fail to exists, even if the society satisfies $P E, A N$ and the indirect utility of each individual is quasi-linear:

Example 5.1.3: Consider the society $\mathcal{E}$, where the set of individuals is given by $N=\{1,2,3,4,5,6,7\}$, and the set of projects is given by $X=\{a, b, c\}$. The indirect utility function $v_{i}(S, x)=$ $|S|+\tilde{v}_{i}(x)$ is quasi-linear for each $i \in N$, and

$$
\begin{array}{r}
\tilde{v}_{1}(a)=6.5, \tilde{v}_{1}(b)=8, \tilde{v}_{1}(c)=4.3, \tilde{v}_{2}(a)=4.3, \tilde{v}_{2}(b)=8.5, \tilde{v}_{2}(c)=6 \\
\tilde{v}_{3}(a)=6, \tilde{v}_{3}(b)=6.3, \tilde{v}_{3}(c)=6.5, \tilde{v}_{4}(a)=8, \tilde{v}-4(b)=0, \tilde{v}_{4}(c)=0 \\
\tilde{v}_{5}(a)=8, \tilde{v}_{5}(b)=0, \tilde{v}_{5}(c)=0, \tilde{v}_{6}(a)=0, \tilde{v}_{6}(b)=0, \tilde{v}_{6}(c)=8 \\
\tilde{v}_{7}(a)=0, \tilde{v}_{7}(b)=0, \tilde{v}_{7}(c)=8 .
\end{array}
$$

Then $\mathcal{E}$ does not admit a $T$-stable outcome. 
The challenge here is to describe the class of societies satisfying $P E$ that admit $T$-stable outcomes. Trivially, it contains all homogeneous societies, where for all $S \subseteq N$, all $i, j \in S$ and all $x, y \in X$, the inequality $v_{i}(S, x) \geq v_{i}(S, x)$ implies $v_{j}(S, x) \geq v_{j}(S, y)$. This extreme case of homogeneity fully exploits the advantages of increasing returns to size and may suggest that a $T$-stable outcome will exist if a degree of homogeneity within the society is large enough. There is no uncontroversial definition of a measure of homogeneity associated to a profile of preferences and we present here the result suggesting that if a unidimensional heterogeneity may indeed yield a $T$-stable outcome.

The result imposes a condition on the profile of individuals preferences which has been called consecutiveness (Greenberg and Weber (1986), (1994)) or intermediate preferences (Demange (1994)). It requires the existence of an ordering of the individuals with respect to their preferences:

Consecutiveness - CONS: Let $\mathcal{E}$ be a society satisfying $A N . \mathcal{E}$ is consecutive if there exists an ordering $\prec$ over $N$ such that for all $i, j, k \in N$, all $x, x^{\prime} \in X$ and all positive integers $r, r^{\prime}$, if $i \prec j \prec k, v_{i}(r, x)<v_{i}\left(r^{\prime}, x^{\prime}\right)$ and $v_{k}(r, x)<v_{k}\left(r^{\prime}, x^{\prime}\right)$ then $v_{j}(r, x)<v_{j}\left(r^{\prime}, x^{\prime}\right)$.

The interpretation of this condition is that if any two individuals prefer to be in a group with $r^{\prime}$ members and policy $x^{\prime}$ rather than in a group with $r$ members and policy $x$, then any intermediate individual (according to the ordering $\prec$ ) must share this choice.

We have the following result, the proof of which is presented in the Appendix:

Proposition 5.1.4: Every society $\mathcal{E}$, satisfying $P E, A N$ and $C O N S$, admits a $T$-stable outcome.

Many alternative versions of Proposition 5.1.4 could be provided. In particular, $A N$ is not essential but the definition of consecutiveness without $\mathrm{AN}$ is more cumbersome. Further, as demonstrated by Demange (1994), the assumption of a complete ordering $\prec$ is not essential as well. Demange proved a version of Proposition 5.1.4, where, instead of $C O N S$, she assumes the existence of a tree $e^{9} \tau$ on $N$ such that for all $x, x^{\prime} \in X$, the $\operatorname{sets}^{10}\left\{i \in N: v_{i}(x)<v_{i}\left(x^{\prime}\right)\right\}$ and

\footnotetext{
${ }^{9}$ A tree $\tau$ on $N$ is a graph on $N$ such that there is a unique path between any two distinct elements of $N$; a subset $S \subseteq N$ is connected with respect to $\tau$ if : $i, j \in S \Rightarrow k \in S$ whenever $k$ is on the path from $i$ to $j$.

${ }^{10}$ In her model, $v_{i}(S, x)$ does not depend on $S$.
} 
$\left\{i \in N: v_{i}(x) \leq v_{i}\left(x^{\prime}\right)\right\}$ are connected with respect to $\tau$.

\section{$5.2 \quad$ Individual $T$-stability}

The discussion in the previous subsection indicates that the notion of $T$-stability, that requires a $T$-attainable outcome to be immune against any group deviation, is quite demanding. It would naturally lead us to attempt to examine a less restrictive concept of stability and we consider a notion of individual $T$-stability, where an outcome has to be immune against individual deviations only. The difference between two concepts of stability is reminiscent of the distinction between the notion of Nash equilibrium and of strong Nash equilibrium (Aumann (1959)).

Let $\mathcal{E}$ be a finite society and consider a pair $\left\{\left(S^{k}, x^{k}\right)\right\}_{1 \leq k \leq K}$, where $\left\{S^{k}\right\}_{1 \leq k \leq K} \in \mathcal{P}$ is a group structure and $x^{k}$ are projects in $X$. Recall that $T$ is a single-valued cost allocation correspondence that determines the individual contributions and allows to focus on groups and projects' selection only. We will introduce the notion of individual T-stability that satisfies the following natural requirements. First, no individual $i$ would prefer staying alone rather than accepting the project $x^{k(i)}$ in the group $S^{k(i)}$. Secondly, no individual $i$ would contemplate joining another coalition $S^{j}$ in the existing partition $\left.P=\left(S^{k}\right)\right\}_{1 \leq k \leq K}$ that chooses the project $x^{j}$. The notion of individual $T$-stability is in the spirit of Nash equilibrium where no individual would rather deviate to one of the existing groups or stay alone:

Definition 5.2.1: A $T$-attainable outcome $\left\{P, x^{k}\right\}_{1 \leq k \leq K}$, where $P=\left\{S^{k}\right\}_{1 \leq k \leq K} \in \mathcal{P}$ is a group structure is called a individually $T$-stable if the following two inequalities hold for for every $i \in N$ :

$$
\text { (i) } v_{i}\left(S^{k(i)}, x^{k(i)}\right) \geq \max _{x \in X} v_{i}(\{i\}, x)
$$

and

$$
\text { (ii) } v_{i}\left(S^{k(i)}, x^{k(i)}\right) \geq \max _{S^{j} \in P} v_{i}\left(S^{j} \bigcup\{i\}, x^{j}\right)
$$

Note we do not impose here any sort of restrictions on the "migration" of individuals from one group to another. We could imagine situations where such deviations, as under contractual 
stability introduced in Drèze and Greenberg (1980), are subject to approval of a fraction of the players in the new group or/and in the old group. Also, the notion of individual $t$-stability is provided here for finite societies. For infinite nonatomic societies, one can consider a modified notion capturing the robustness of a $T$-attainable outcome to deviations by coalitions of an arbitrarily small size (see subsection 5.3).

It is easy to verify that, under $P E$, the notion of individual $t$-stability is weaker than $T$ stability. The following remark is a corollary of the result in Le Breton and Weber (2003b):

Remark 5.2.2: Every $T$-stable outcome in a society $\mathcal{E}$ that satisfies $P E$ is individually $T$-stable.

The following example (Konishi, Le Breton and Weber (1997b)) shows that there exists a society, satisfying $A N$ and $P E$, but does not admit even an individually stable $T$-outcome.

Example 5.2.3: Consider the society $\mathcal{E}$, where the set of individuals $N$ and projects $X$ are given by $N=\{1,2,3\}$, and $X=\{a, b, c\}$, respectively. (By Propositions 5.1.1 and 5.1.2, this is the minimal size of $N$ and $X$ that may generate an absence of stability.) The society $\mathcal{E}$ satisfies $A N$ and $P E$, and the individuals' utility functions of individuals satisfy the following inequalities:

$$
\begin{aligned}
& v_{1}(2, b)>v_{1}(2, a)>v_{1}(1, a)>v_{1}(1, b)>v_{1}(3, c) \\
& \left.v_{(} 2, c\right)>v_{2}(2, b)>v_{2}(1, b)>v_{2}(1, c)>v_{2}(3, a) \\
& v_{3}(2, a)>v_{3}(2, c)>v_{3}(1, c)>v_{3}(1, a)>v_{3}(3, b) .
\end{aligned}
$$

Then $\mathcal{E}$ does not admit an individually $T$-stable outcome.

This rather simple example shows that one has to impose much more demanding conditions to guarantee the existence of an individually $T$-stable outcomes in societies with more than two individuals and two projects. The following result (Konishi, Le Breton and Weber (1997a)) demonstrates that the more demanding condition is sufficient for the existence of an individually $T$-stable outcome. It is also shown that the result is tight in the sense that relaxing any of the assumptions would deny the existence result. 
Proposition 5.2.4: Let $\mathcal{E}$ be a society satisfying $P E$ and $A N$. Moreover, the utility functions ${ }^{11}$ of each $i \in N$ are quasi-linear: $v_{i}(S, x)=|S|+\tilde{v}_{i}(x)$. Then the society $\mathcal{E}$ admits an individually $T$-stable outcome.

The proof of this proposition is quite instructive and proceeds by constructing a real-valued function on the set of $n$-tuples $\left(x^{1}, \ldots, x^{n}\right)$ of individuals' project choices. It turns out that every local maximum of this function (the existence of which is guaranteed by the finiteness of the domain) yields an individually $T$-stable outcome. This method of proof of is similar to the one used by Rosenthal (1973), who introduced the class of "potential games" studied further by Monderer and Shapley (1996).

\subsection{Equal Cost Sharing Rule}

We examine now a finite society with a single-valued cost allocation correspondence $T^{E}$, where each coalition can use only equal cost sharing rule to finance the cost of public projects. We assume that for every project $x \in X$ the cost $C(S, x)$ is independent of group $S$ and that the utility functions of all individuals are quasi-linear. Then the indirect utility of an individual $i$ who belongs to group $S$ choosing project $x$, is given by

$$
v_{i}(S, x)=\tilde{v}_{i}(x)+w(i)-\frac{C(x)}{|S|}
$$

We assume that the initial endowment of every individual is sufficiently large to cover contributions to all projects so, without further confusion, we will simply delete the term $w(i)$.

The existence of an individually $T^{E}$-stable outcome in this framework can be derived from Proposition 5.2.4. It is interesting to note, however, that individually $T^{E}$-stable outcomes can be inefficient. The following example (Konishi, Le Breton and Weber (1998)) shows that there is a society with several $T^{E}$-stable outcomes such of which are Pareto inferior to others.

Example 5.3.1: Consider a society $\mathcal{E}$, with two individuals, $N=\{1,2\}$, and three public

\footnotetext{
${ }^{11}$ See Konishi and Fishburn (1996) for the assumptions needed for this utility representation.
} 
projects, $X=\{a, b, c\}$. Let utilities of individuals be given by:

$$
\tilde{v}_{1}(a)=30, \tilde{v}_{1}(b)=0, \tilde{v}_{1}(c)=0, \tilde{v}_{2}(a)=0, \tilde{v}_{2}(b)=0, \tilde{v}_{2}(c)=25
$$

and the project costs are $C(a)=C(b)=C(c)=20$.

This society has two individually $T^{E}$-stable outcomes, one where individual 1 choose project $a$ and individual 2 chooses project $b$ and the other where both choose project $c$. The utility vectors associated with two outcomes are $(10,-20)$ and $(15,15)$, respectively. Thus, the first is Pareto inferior to the second.

Example 5.3.1 illustrates a conventional inefficiency arising from a pure coordination problem. To evaluate the potential scope of inefficiency of $T$-stable outcomes, consider the following much weaker efficiency requirement:

Definition 5.3.2: A $T^{E}$-attainable outcome $\left\{P, x^{k}\right\}_{1 \leq k \leq K}$, where $P=\left\{S^{k}\right\}_{1 \leq k \leq K}$ is a partition of $N, x^{k} \in X$ are public projects chosen by $S^{k}$, is intra-group efficient if there is no $S^{k} \in P$ and $y \in X$ such that $\tilde{v}_{i}(y)-\frac{C(y)}{\left|S^{k}\right|} \geq \tilde{v}_{i}(x)-\frac{C(x)}{\left|S^{k}\right|}$ for all $i \in S^{k}$ with a strict inequality for, at least, one $i$.

Intra-group efficiency rules out arrangements across groups and does not allow for their break up. It simply requires that no group in $P$ can find an alternative public project preferred to the chosen one by all members of the group. The following example, also taken from Konishi, Le Breton and Weber (1998), demonstrates that intra-group efficient individually $T^{E}$-stable outcomes may fail to exist. This obviously implies that $T^{E}$-stable outcomes may fail to exist as well.

Example 5.3.3: Consider a society $\mathcal{E}$, with five individuals, $N=\{1,2,3,4,5\}$, and four public projects, $X=\{a, b, c, d\}$. Let utilities of individuals be given by:

$$
\begin{gathered}
\tilde{v}_{1}(a)=10025, \tilde{v}_{1}(b)=10110, \tilde{v}_{1}(c)=0, \tilde{v}_{1}(d)=10061, \\
\tilde{v}_{2}(a)=10026, \tilde{v}_{2}(b)=10089.8, \tilde{v}_{2}(c)=0, \tilde{v}_{2}(d)=10030, \\
\tilde{v}_{3}(a)=10030, \tilde{v}_{3}(b)=10120, \tilde{v}_{3}(c)=9900, \tilde{v}_{3}(d)=9900,
\end{gathered}
$$




$$
\begin{gathered}
\tilde{v}_{4}(a)=10000, \tilde{v}_{4}(b)=10240, \tilde{v}_{4}(c)=10200, \tilde{v}_{4}(d)=9900 \\
\tilde{v}_{5}(a)=9900, \tilde{v}_{5}(b)=9900, \tilde{v}_{5}(c)=10090, \tilde{v}_{5}(d)=10061
\end{gathered}
$$

and the project costs are $C(a)=50, C(b)=240, C(c)=90$ and $C(d)=60$.

This society has a unique individually $T^{E}$-stable outcome where individuals $1,2,3$ choose $a$, while 4 and 5 choose $c$. However, this outcome is not intra-group efficient as individuals $1,2,3$ would be better off by jointly switching to project $b$.

We now consider the infinite nonatomic framework examined in Section 4. In doing so we will modify the notion of an individually $T$-stable outcome to take into account the deviation of arbitrarily small coalitions (see also Jehiel and Scotchmer (2001), Le Breton and Weber (2003c)):

Definition 5.3.4: Let $\mathcal{E}$ be an atomic society and $z=\left\{\left(S^{k}, x^{k}\right)\right\}_{1 \leq k \leq K}$ is a $T^{E}$-attainable outcome. Let $u$ be the utility vector associated with $z$, where for every $i \in N$ :

$$
u_{i}=-\left|i-x^{k(i)}\right|-\frac{C\left(S^{k(i)}, x^{k(i)}\right)}{\mu\left(S^{k(i)}\right)} .
$$

$z$ is called locally $T$-stable if there exists $\delta>0$ such that there is no coalition $S$ with $\mu(S) \leq \delta$ and $x \in X$ such that

$$
u_{i}(S, x)=-|i-x|-\frac{C(S, x)}{\mu(S)}>u_{i}
$$

for all $i \in S$.

By this definition, a $T^{E}$-attainable outcome is locally $T^{E}$-stable if there is no coalition of positive but arbitrary small measure for which it would be profitable to deviate. It is less demanding than $T^{E}$-stability as the test against coalitional deviations applies only to "small" coalitions. Obviously, the set of locally stable $T^{E}$-outcomes is smaller than the set of individually $T^{E}$-stable outcomes but is larger than the set of $T^{E}$-stable outcomes.

The immediate application of the last stability notion is:

Proposition 5.3.5: Let the distribution of individuals' location be uniform on $X$. If a $T^{E_{-}}$ attainable outcome $z=\left\{\left(S^{k}, x^{k}\right)\right\}_{1 \leq k \leq K}$ is locally $T^{E}$-stable than all groups $S^{k}$ are of equal size and their number $K$ is smaller than $\frac{1}{\sqrt{2 c}}$. 
Alesina and Spolaore (1997) consider a version of contractual stability where a new group can be created or an existing group can be eliminated if the change is approved by majority vote in each of the groups affected by that decision. Under the assumption of the uniform distribution, they show that a group structure consisting of groups of equal size is stable in that sense if and only if the number of groups is the largest integer smaller than $\frac{1}{\sqrt{2 c}}$. They also show that if secession threats are limited to subgroups of the group structure, then a group structure consisting of groups of equal size is stable if and only if the number of groups is larger than $\frac{1}{(\sqrt{6}+2) \sqrt{c}}$.

We now turn to the finite variant of the model presented in Section 4 where $X \subseteq \Re$ and $v_{i}(x, m(i))=-d(i, x)+m(i)$ for all $i \in N$. As we indicated above, $x$ is interpreted as a location decision and $d(i, x)$ as the transportation cost incurred by $i$ when she "consumes" a public project located at $x$. If the incomes of all individuals are the same, the existence of an individually $T^{E_{-}}$ stable outcome follows from Proposition 5.2.4. Let us now examine the existence of $T^{E}$-stable outcomes. Let $\theta(i)$ denotes the location of individual $i$ on the real line. The following result follows from Proposition 5.1.4:

Proposition 5.3.6: If $d(i, x)=g(|\theta(i)-x|)$ where $d: \Re_{+} \rightarrow \Re_{+}$is increasing and convex, then there exists a $T^{E}$-stable outcome.

In this proposition, it is assumed that the transportation cost of any individual is an increasing function of her distance to the public project and that this function is the same for all individuals. In particular, the transportation cost function of any individual is symmetric with respect to her peak. It is easy to see that under the assumptions of this proposition, any $T^{E}$-stable outcome is stratified in the sense that it each group is an interval according to the order $\prec$. The following example shows that, in general, this is not the case:

Example 5.3.7: Consider a society with four individuals, $N=\{1,2,3,4\}$, with their locations $\theta(1)<\theta(2)<\theta(3)<\theta(4)$ are given by $\theta(2)=\theta(1)+\gamma, \theta(3)=\theta(1)+2 \gamma, \theta(4)=\theta(1)+3 \gamma$, 
where $\gamma$ is a positive parameter. The transportation costs are determined as follows:

$$
\begin{aligned}
& d(1, x)=\left\{\begin{array}{c}
\frac{x-\theta(1)}{\varepsilon} \text { if } x>\theta(1) \\
\varepsilon(\theta(1)-x) \text { if } x<\theta(1)
\end{array}\right. \\
& d(2, x)=\left\{\begin{array}{c}
\varepsilon(x-\theta(2)) \text { if } x>\theta(2) \\
\frac{\theta(2)-x}{\varepsilon} \text { if } x<\theta(2)
\end{array}\right. \\
& d(3, x)=\left\{\begin{array}{c}
\frac{x-\theta(3)}{\varepsilon} \text { if } x>\theta(3) \\
\varepsilon(\theta(3)-x) \text { if } x<\theta(3)
\end{array}\right. \\
& d(4, x)=\left\{\begin{array}{c}
\varepsilon(x-\theta(4)) \text { if } x>\theta(4) \\
\frac{\theta(4)-x}{\varepsilon} \text { if } x<\theta(4)
\end{array}\right.
\end{aligned}
$$

where $0<\varepsilon<\min \left[\frac{2 \gamma}{3 c}, \frac{c}{4 \gamma}\right]$. Finally, the project costs $C(x)=c$ for all $x \in X$. Then for every $T^{E}$-stable outcome $\left\{P, x^{1}, \ldots, x^{K}\right\}$ the partition $P$ consists of two pairs, $\{1,3\}$ and $\{2,4\}$.

First, we show that in a $T^{E}$-stable outcome, the pairs of individuals 1,2 and 3,4 cannot be in the same group. Suppose, in negation, that 1 and 2 are in the same group $S$ with a public project located in $x$. Then either $d(1, x) \geq \frac{\gamma}{2 \varepsilon}$ or $d(2, x) \geq \frac{\gamma}{2 \varepsilon}$. Therefore either $v_{1}(S, x) \leq-\frac{\gamma}{2 \varepsilon}-\frac{c}{4}$ or $v_{2}(S, x) \leq-\frac{\gamma}{2 \varepsilon}-\frac{c}{4}$. On the other hand, $v_{1}(\{1\}, \theta(1))=-c$ and

$v_{2}(\{2\}, \theta(2))=-c$. Thus, if $\varepsilon<\frac{2 d}{3 c}$, then either $v_{1}(S, x)<v_{1}(\theta(1),\{1\})$ or $v_{2}(S, x)<$ $v_{2}(\theta(2),\{2\})$, contradicting our assumption that $S$ is part of a $T^{E}$-stable outcome. A similar argument applies to the pairs $\{3,4\},\{1,4\},\{2,3\}$.

To conclude it remains to show that 1 and 3 prefer to be together rather than staying alone. But $v_{1}(\{1,3\}, \theta(1))=-\frac{c}{2}$ and $v_{3}(\{1,3\}, \theta(1))=-2 \varepsilon \gamma-\frac{c}{2}$. Thus, $v_{1}(\{1,3\}, \theta(1))>$ $v_{1}(\{1\}, \theta(1))$ and $v_{3}(\{1,3\}, \theta(1))>v_{3}(\theta(3),\{3\})$ whenever $\varepsilon<\frac{c}{4 \gamma}$.

\section{$6 \quad$ Appendix}

Proof of Proposition 5.1.4: Let $E$ be a finite society satisfying $P E, A N$ and $C O N S$. The proof of the existence of a $T$-stable outcome will proceed in nine steps.

Step 1: Construction of an auxiliary cooperative game with a nonempty core.

Let $\mathcal{C}$ be the family of subsets of $N$ which are intervals with respect to the ordering $\prec$. We 
associate a cooperative game in characteristic form $\widehat{v}_{\mathcal{C}}$ to $E$ as follows

$$
\widehat{v}_{\mathcal{C}}(S)=\cup_{P_{S} \in \mathcal{P}_{\mathcal{C}}(S)} \cap_{T \in P_{S}} v(T)
$$

where $\mathcal{P}_{\mathcal{C}}(S)$ denotes the set of partitions of $S$ into subsets of $\mathcal{C}$. The game $\widehat{v}$ describes the set of payoffs feasible for every coalition assuming it can be partitioned into intervals.

As demonstrated ${ }^{12}$ by Greenberg and Weber (1986), the cooperative game $\widehat{v}_{\mathcal{C}}$ has a nonempty core. Let a pair $\left\{\left(x^{k}, S^{k}\right)\right\}_{1 \leq k \leq K}$, where $P=\left\{S^{k}\right\}_{1 \leq k \leq K} \in \mathcal{P}_{\mathcal{C}}$ is a group structure (consisting of intervals) and $x^{k} \in X$ for all $k=1, \ldots, K$, be an outcome corresponding to the utility vector $u$ that belongs to the core of $\widehat{v}_{\mathcal{C}}$. Let $u_{i}=u_{i}\left(x^{k(i)}, r(i)\right)$ where $i \in S^{k}(i)$ and $r(i) \equiv\left|S^{k(i)}\right|$. The groups are labeled from left to right i.e. $k<k^{\prime}$ if and only if $i \prec j$ for all $i \in S^{k}, j \in S^{k^{\prime}}$ and we denote by $j(k)$ the minimal number, according to $\prec$, in $S^{k}$.

We will use the term "to migrate" for an individual who would rather prefer another coalition within the existing partition and the project chosen there over the coalition she is currently in.

Step 2: For all $k=2, \ldots, K, j(k)$ would not migrate to $S^{k-1}$.

Assume, to the contrary, that for some $k, j(k)$ would migrate to $S^{k-1}$, i.e., $u_{j(k)}\left(x^{k-1}, r(j(k)-\right.$ $1)+1)>u_{j(k)}\left(x^{k}, r(j(k))\right)$. By $P E, u_{i}\left(x^{k-1}, r(j(k)-1)+1\right)>u_{i}\left(x^{k-1}, r(j(k)-1)\right)$, contradicting the assumption that the utility vector $u$ belongs to the core of $\widehat{v}_{\mathcal{C}}$ as $S^{k-1} \cup\{j(k)\} \in \mathcal{C}$.

Step 3: For all $k=1, \ldots, K-1, j(k)-1$ would not migrate to $S^{k}$. The argument is identical to that in Step 2.

Step 4: For all $k=2, \ldots, K$ and all $i \in S^{k}, i$ would not migrate to $S^{k-1}$.

Suppose, on the contrary, that $u_{i}\left(x^{k-1}, r(j(k)-1)+1\right)>u_{i}\left(x^{k}, r(j(k))\right)$ for some $i \in S^{k}$. Since, from Step $3, u_{j(k)-1}\left(x^{k-1}, r(j(k)-1)\right) \geq u_{j(k)-1}\left(x^{k}, r(j(k))+1\right), P E$ yields $u_{j(k)-1}\left(x^{k-1}, r(j(k)-\right.$ $1)+1) \geq u_{j(k)-1}\left(x^{k}, r(j(k))\right)$. Thus, by $C O N S$, we have $u_{j(k)}\left(x^{k-1}, r(j(k)-1)+1\right)>u_{j(k)}\left(x^{k}, r(j(k))\right)$, contradicting the assertion in Step 2.

Step 5: For all $k=1, \ldots, K-1$ and all $i \in S^{k}, i$ would not migrate to $S^{k+1}$. The proof is identical to that in Step 4.

\footnotetext{
${ }^{12} \mathrm{~A}$ general characterization of the family of coalitions $\mathcal{C}$ for which the game $\widehat{v}_{\mathcal{C}}$ has a nonempty core is provided in Le Breton, Owen and Weber (1992).
} 
Step 6: For all $k=3, \ldots, K$ and all $i \in S^{k}, i$ would not migrate to $S^{k-2}$.

Suppose, in negation, that $\left.u_{i}\left(x^{k-2}, r(j(k-1)-1)\right)+1\right)>u_{i}\left(x^{k}, r(j(k))\right)$ for some $i \in S^{k}$. Since from Step $4 u_{i}\left(x^{k}, r(j(k))\right) \geq u_{i}\left(x^{k-1}, r(j(k)-1)+1\right), P E$ implies that $u_{i}\left(x^{k-2}, r(j(k-1)-\right.$ $1))+1)>u_{i}\left(x^{k-1}, r(j(k)-1)\right)$. On the other hand, from Step 3 and $P E, u_{j(k-1)-1}\left(x^{k-2}, r(j(k-\right.$ $1)-1))+1)>u_{j(k-1)-1}\left(x^{k-1}, r(j(k)-1)\right)$. Then, by $\left.C O N S, u_{j}\left(x^{k-2}, r(j(k-1)-1)\right)+1\right)>$ $u_{j}\left(x^{k-1}, r(j(k)-1)\right)$ for all $j \in S^{k-1}$, contradicting the assertion in Step 4.

Step 7: For all $k=1, \ldots, K-2$ and all $i \in S^{k}, i$ would not migrate to $S^{k+2}$. The proof is identical to the proof of Step 6.

Steps 1-7, combined with a straightforward induction argument, imply:

Step 8: $\left\{S^{k}, x^{k}\right\}_{1 \leq k \leq K}$ is an individually $T$-stable outcome.

Step 9: $\left\{S^{k}, x^{k}\right\}_{1 \leq k \leq K}$ is a $T$-stable outcome.

Suppose, on the contrary, that there exists $S \subseteq N$ and $x \in X$ such that $u_{i}(x, S)>u_{i}\left(x^{k(i)}, r(i)\right)$ for all $i \in S$.

Since $S \notin \mathcal{C}$, there exists $i, l \in S, j \notin S$ such that $i \prec j \prec l$. If $j \notin S^{k(i)}$, we deduce from Step 8 and $P E$ that $u_{i}(x, S) \geq u_{i}\left(x^{k(j)}, r(j)+1\right)>u_{i}\left(x^{k(j)}, r(j)\right)$ and $u_{l}(x, S) \geq u_{l}\left(x^{k(j)}, r(j)+1\right)$.

If $j \in S^{k(i)}$, then $x^{k(i)}=x^{k(j)}$ and, trivially, $u_{i}(x, S)>u_{i}\left(x^{k(j)}, r(j)\right)$. A similar argument for $l$ leads to $u_{l}(x, S) \geq u_{l}\left(x^{k(j)}, r(j)\right)$.

$C O N S$ implies that $u_{j}(x, S) \geq u_{j}\left(x^{k(j)}, r(j)\right)$. If $S \cup\{j\} \in \mathcal{C}$, we contradict our assumption that the utility vector $u$ is in the core of $\widehat{v}_{\mathcal{C}}$. If not, we repeat the argument with group $S \cup\{j\}$. Since $\mathrm{N}$ is finite, the process will be terminated after a finite number of iterations.

\section{References}

Ahmad, E. and J. Craig (1997) "Intergovernmental Transfers", in Fiscal Federalism in Theory and Practice, T. Ter-Minassian, ed., International Monetary Fund, Washington D.C.

Alesina, A. and E. Spolaore (1997) "On the number and size of nations", Quarterly Journal of Economics 113, 1027-1056.

Arthur, W.B. (1989) “Competing Technologies, Increasing Returns, and Lock-in by Historical 
Events", Economic Journal 99, 116-131.

Aumann, R.J. (1959) "Acceptable points in general cooperative $n$-person games", in Contributions to the Theory of Games, Vol. IV, Tucker, A.W. and R.D. Luce, eds., Princeton University Press, Princeton.

Aumann, R.J. and J. Drèze (1974) "Cooperative games with coalition structure", International Journal of Game Theory 3, 217-237.

Bagnoli, M. and T. Bergstrom (1989) "Log-Concave Probability and its Applications", University of Michigan Discussion Paper.

Caplin, A. and B. Nalebuff (1991) "Aggregation and Social Choice: A Mean Voter Theorem", Econometrica 59, 1-24.

Cremer, H., De Kerchove, A.M. and J. Thisse (1985) "An economic theory of public facilities in space", Mathematical Social Sciences 9, 249-262.

Conley, J.P. and M.H. Wooders (1997) "Anonymous pricing in Tiebout economies and economies with clubs", in Topics in Public Finance, Pines, D., Sadka, E. and Y. Zilcha, eds., Cambridge University Press, Cambridge.

Demange, G. (1994) "Intermediate preferences and stable coalition structures", Journal of Mathematical Economics 23, 45-58.

Drèze, J. and J. Greenberg (1980) "Hedonic coalitions: optimality and stability", Econometrica, 48, 987-1003.

Ellickson, B, Grodal, B., Scotchmer, S. and W.R. Zame (1999) "Clubs and the market", Econometrica 67, 1185-1217.

Farrell, J. and G. Saloner (1985) "Standardization, Compatibility, and Innovation", Rand Journal of Economics 16, 70-83.

Farrell, J. and G. Saloner (1988) "Coordination through Committees and Markets", Rand Journal of Economics 19, 235-252.

Greenberg, J. and S. Weber (1986) "Strong Tiebout equilibrium under restricted preferences domain", Journal of Economic Theory 38, 101-117. 
Greenberg, J. and S. Weber (1993) "Stable coalition structures with a unidimensional set of alternatives", Journal of Economic Theory 60, 62-82.

Greenberg, J. and S. Weber (1994) "Stable coalition structures in consecutive games", in Frontiers in Game Theory, Binmore, K., Kirman, A. and P. Tani, eds., MIT Press, Cambridge.

Guesnerie, R. and C. Oddou (1979) "On economic games which are not necessarily superadditive", Economic Letters 3, 301-306.

Guesnerie, R. and C. Oddou (1981) "Second best taxation as a game", Journal of Economic Theory 25, 67-91.

Haimanko, O., Le Breton, M. and S. Weber (2003a) “Transfers in a polarized country: bridging the gap between efficiency and stability", Journal of Public Economics, forthcoming.

Haimanko, O., Le Breton, M. and S. Weber (2003b) "Voluntary formation of communities for the provision of public projects", Journal of Economic Theory, forthcoming.

Jehiel, P. and S. Scotchmer (2001) "Constitutional rules of exclusion in jurisdiction formation", Review of Economic Studies 68, 393-413.

Konishi, H. and P.C. Fishburn (1996) "Quasi-linear utility in a discrete choice model", Economic Letters 51, 197-200.

Konishi, H., Le Breton, M. and S. Weber (1997a) "Pure strategy Nash equilibria in a group formation game with positive externalities", Games and Economic Behavior 21, 161-182.

Konishi, H., Le Breton, M. and S. Weber (1997b) "Group formation in games without spillovers: a noncooperative game-theoretical approach", in New Directions in the Economic Theory of the Environment, Carraro, C. and D. Siniscalco, eds., Cambridge University Press, Cambridge.

Konishi, H., Le Breton, M. and S. Weber (1998) "Equilibrium in a finite local public goods economy", Journal of Economic Theory 79, 224-244.

Le Breton, M., Owen, G. and S. Weber (1992) "Strongly balanced cooperative games", International Journal of Game Theory 20, 419-427.

Le Breton, M. and S. Weber (2003a) "The art of making everybody happy: how to prevent a 
secession", IMF Stuff Papers, forthcoming.

Le Breton, M. and S. Weber (2003b) "Stable partitions in a model with group-dependent feasible sets", Southern Methodist University and University of Toulouse, Mimeo.

Le Breton, M. and S. Weber (2003c) "Group formation in strategic environments without widespread externalities", Southern Methodist University and University of Toulouse, Mimeo.

Mas-Collel, A. (1980) "Efficiency and decentralization in the pure theory of public goods", Quarterly Journal of Economics 94, 625-641.

Monderer, D. and L.S. Shapley (1996) "Potential games", Games and Economic Behavior 14, 124-143.

Rosenthal, R.W. (1973) "A class of games possessing a pure-strategy Nash equilibrium", International Journal of Game Theory 2, 65-67.

Schmeidler, D. (1973) "Equilibrium points in nonatomic games", Journal of Statistical Physics, 7, 295-300.

Ter-Minassian, T., ed. (1997) "Fiscal Federalism in Theory and Practice", International Monetary Fund, Washington, D.C.

Tirole, J. (1988) Industrial Organization, MIT Press, Cambridge.

Weber, S. (1992) "On Hierarchical Spatial Competition", Review of Economic Studies 59, 407-425.

Wooders, M.H. (1978) "Equilibria, the core and jurisdiction structures in economies with a local public good", Journal of Economic Theory, 18, 328-348.

Wooders, M.H. (1980) "The Tiebout hypothesis: near optimality in local public good economies", Econometrica 48, 1467-1486.

Wooders, M.H. (1988) "Stability of jurisdictions structures in economies with local public goods", Mathematical Social Sciences 15, 29-49. 